\title{
Neurophysiological Age Differences During Task-Performance in a Stereoscopic Virtual Environment
}

\author{
Ralph Mager, ${ }^{1,5}$ Oliver Stefani, ${ }^{1}$ Isabelle Angehrn, ${ }^{1}$ Franz Mueller-Spahn, ${ }^{1}$ \\ Evangelos Bekiaris, ${ }^{2}$ Brenda K. Wiederhold, ${ }^{3}$ Hubert Sulzenbacher, ${ }^{4}$ \\ and Alex H. Bullinger ${ }^{1}$
}

In today's society, there is an increasing number of workplaces in virtual environments (VE). But, there are only a few reports dealing with occupational health issues or age effects. The question arises how VR generally interferes with cognitive processes. This interference might have relevant implications for workability and work-efficiency in virtual environments. Event-related potentials are known to reflect different stages of stimulus reception, evaluation, and response. We have established an electroencephalographic (EEG) monitoring, focussing on event-related potentials (N100; mismatch negativity, i.e., MMN) to obtain access to attention dependent and pre-attentive processing of sensory stimuli applied in VE. The MMN is known to be correlated with the ability of subjects to react to an unexpected event. The aim of the present study was to investigate cognitive responses to distracting auditory stimuli in two different age groups in a virtual environment (VE) and in a real environment ("real reality"), and to compare characteristic neurophysiological response patterns. Data show that stimulus detection as given by the N100 amplitude and latency does not differ in both age groups and task conditions. In contrast, the pre-attentive processing as given by the MMN is altered in the VR such as the non-VR condition in an age-related manner. A relevant finding of the present study was that the age related differences seen in the non-VR condition were not strengthened in VR.

KEY WORDS: virtual reality; ERP (event related potential); N100; EEG (electroencephalography); MMN (mismatch negativity).

\footnotetext{
${ }^{1}$ COAT-Basel/UPKBS, Department of Psychiatry, University of Basel, Switzerland.

${ }^{2}$ CERTH/HIT, Centre for Research \& Technology Hellas, Athens, Greece.

${ }^{3}$ Virtual Reality Medical Center, San Diego, USA.

${ }^{4}$ Psychiatric Clinic, University of Innsbruck, Innsbruck, Austria.

${ }^{5}$ Address all correspondence to Dr. Ralph Mager, Director Research and Development, COAT-Basel/UPKBS, Center of Applied Technologies in Neuroscience, Wilhelm Klein-Strasse 27, 4025 Basel, Switzerland; e-mail: ram@coat-basel.com.
} 


\section{INTRODUCTION}

Human aging is a complex process that can be considered to start at different ages depending on the domain of cognition. Age-related declines are proposed to partially result from an inability to inhibit the influence of task-irrelevant information (Hasher \& Zacks, 1988). These age-related changes of performance are already detectable in middle aged people still engaged in the working process (Powers, 1994). In virtual environments (VE), subjects underlie a broad artificial sensory stimulation that might interfere with cognitive processing in an age-dependent manner. Thus, one hypothesis would be that age-dependent differences in cognitive processing in non-VE tasks are strengthened in VE tasks.

One experimental technique that has been successfully applied to studying age-related changes in cognitive processes and their neural correlates is the event-related potential (ERP). ERPs provide a direct noninvasive measure of the temporal electrophysiological changes that occur during central processing. In the present report N100 and MMN were used as parameters. The N100 is reported to reflect stimulus detection in the auditory system in an attention dependent manner (Parasuraman \& Beatty, 1980; Näätänen \& Picton, 1987). The mismatch negativity is an auditory ERP component that has proved to be a valuable parameter for elucidating pre-attentive auditory processing (Tiitinen, May, Reinikainen, Näätänen, 1994). Therefore, in the present study ERPs were used to compare auditorycognitive processing in VR and non-VR conditions and to look for age-related differences.

\section{MATERIAL AND METHODS}

ERPs were recorded in a group of 45 shift workers with a similar level of education. Two different age groups were formed: Group 1: mean age 24.7 years $(n=25)$ and Group 2: mean age 46.0 years $(n=20)$.

Mismatch-negativity (MMN) was chosen as a parameter.

Task 1: A working task ("Tower of Hanoi") was performed in a fully immersive and stereoscopic virtual environment (Fig. 1).

Task 2: A comparable working task was performed in a real environment.

Two additional reading sessions were implemented as control. A passive auditory oddball paradigm was employed during all sessions except a final-sleep pressure trial.

\section{Virtual Reality Setup}

A three-segmented powerwall (manufactured by ICIDO $^{\circledR}$, Germany) was used to present the virtual environment. The powerwall is driven by a cluster of 8 PCs using LINUX $^{\circledR}$ as the operating system. Tracking was done by wireless optical tracking (system manufactured by $\mathrm{ART}^{\circledR}$, Germany) while as an input device, a wireless tracked sphere (manufactured by ICIDO ${ }^{\circledR}$, Germany, also) was used (Fig. 2).

A 32-channel EEG was installed, fitting persons with an electrode cap and data were acquired during the complete exposure. Electrodes were referenced to the connected ear lobes. Two different tones were applied: a standard tone $(500 \mathrm{~Hz})$ and a deviant rare tone $(550 \mathrm{~Hz})$. Stimulation frequency was $1 \mathrm{~Hz}$ during the tasks. Two blocks of 250 stimuli were applied. Tone duration was $125 \mathrm{~ms}$. 


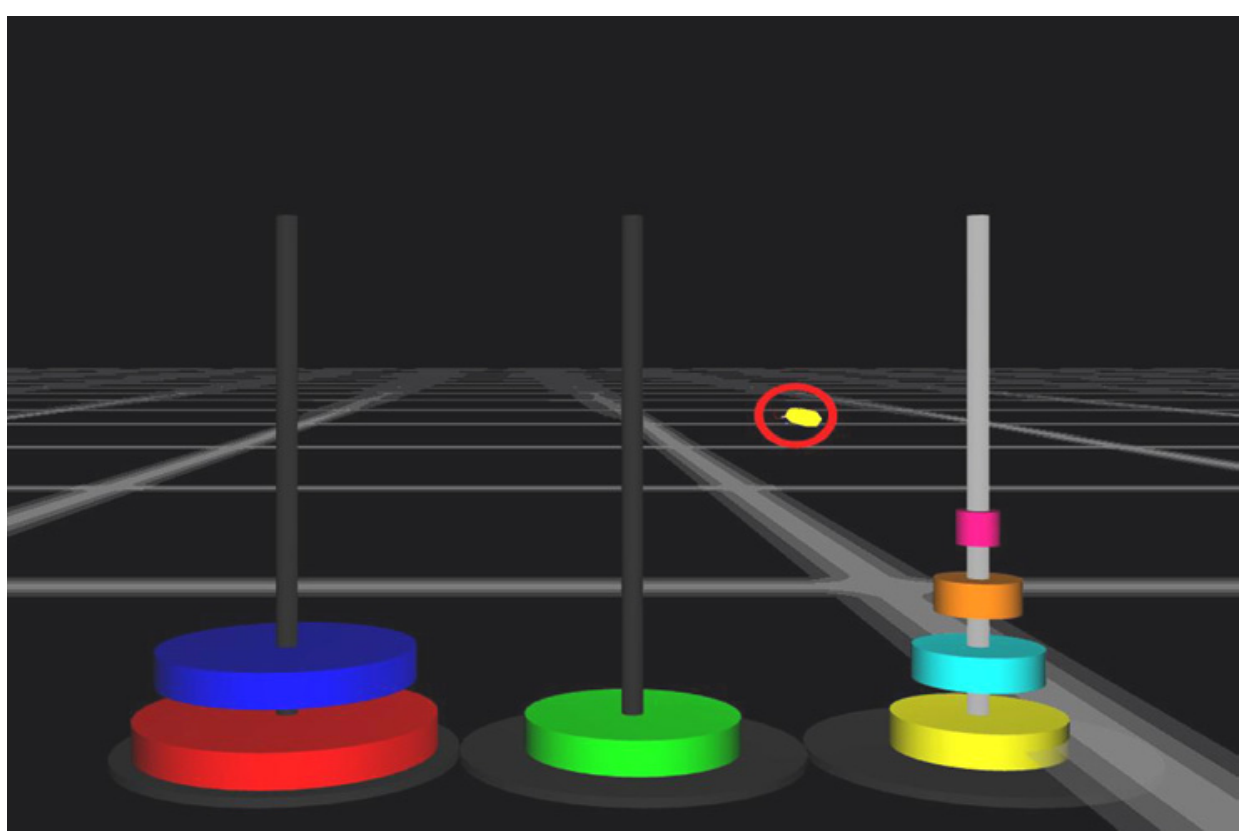

Fig. 1. Screenshot from virtual environment representing the "Tower of Hanoi" used in this experiment.

Data analysis was performed offline. ERP amplitudes and latencies were calculated for different age groups. N100 was identified as a negative peak within 70-110 ms from stimulus onset. Difference waveforms for assessment of the MMN were calculated for each subject by subtracting the averaged waveforms elicited by standards from those elicited by deviant stimuli after re-referencing to linked earlobes. Grand averages of standard and difference waveforms for each age group were constructed for illustrative purposes. Peak latency was determined as the most negative peak in a window between 90 and $250 \mathrm{~ms}$. MMN during the working task in VE was compared with the reality-task condition.

Data was analyzed using descriptive statistical methods. Two-tailed, one-way between subjects, ANOVAs were used to carry out significant differences between the two age groups or between task conditions at every time of measurement. Increase or decrease of physiological values relating to baseline were analyzed using paired $t$-tests.

\section{RESULTS}

\section{Comparison: "VR" Condition Versus "Non-VR" Condition}

The goal of data analysis was to compare the processing of task irrelevant tones (frequent or rare) during the non-VR condition and the VR condition for both age groups. The question behind this paradigm was to evaluate how the age groups, process distracting potentially important (safety aspect) stimuli under both conditions. In Fig. 3, the averaged responses (young, $n=25$; elderly, $n=20$ ) to frequent nontask-relevant tones 


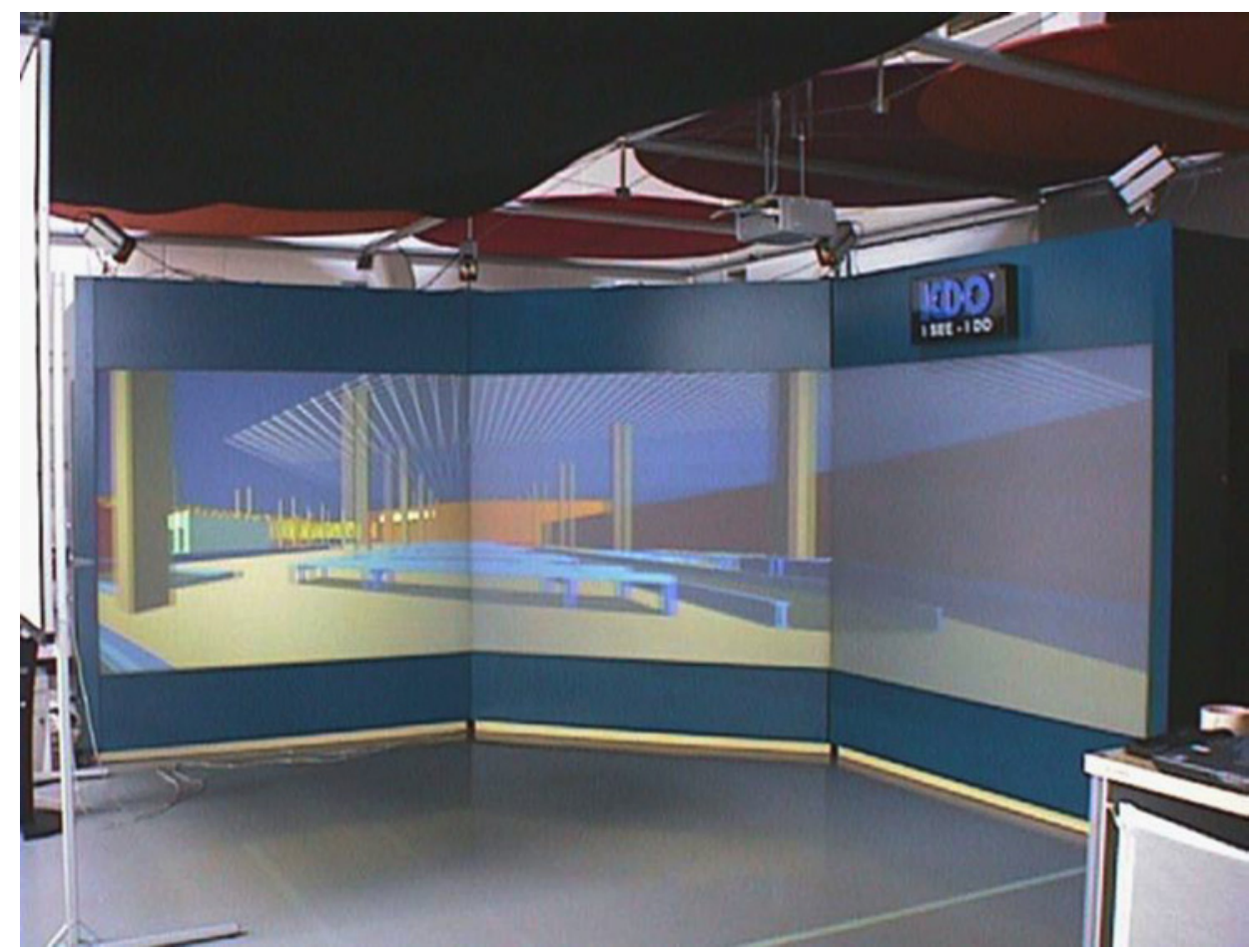

Fig. 2. Three-segmented powerwall used for this experiment.

are illustrated for both age groups under non-VR conditions (Condition I) and under VR conditions (Condition II). In Condition I, the comparison of the N100 potential did not reveal age differences with respect to amplitude or latency (latency Fz: young, mean $=88.8 \pm 8.16 \mathrm{~ms}$; old, mean $=89.33 \pm 5.25 \mathrm{~ms} ; F_{1,44}=0.066, p=0.8$; amplitude Fz: young, mean $=-3.91 \pm 2.05 \mu \mathrm{V}$; old, mean $=-4.40 \pm 1.34 \mu \mathrm{V} ; F_{1,44}=0.88$,
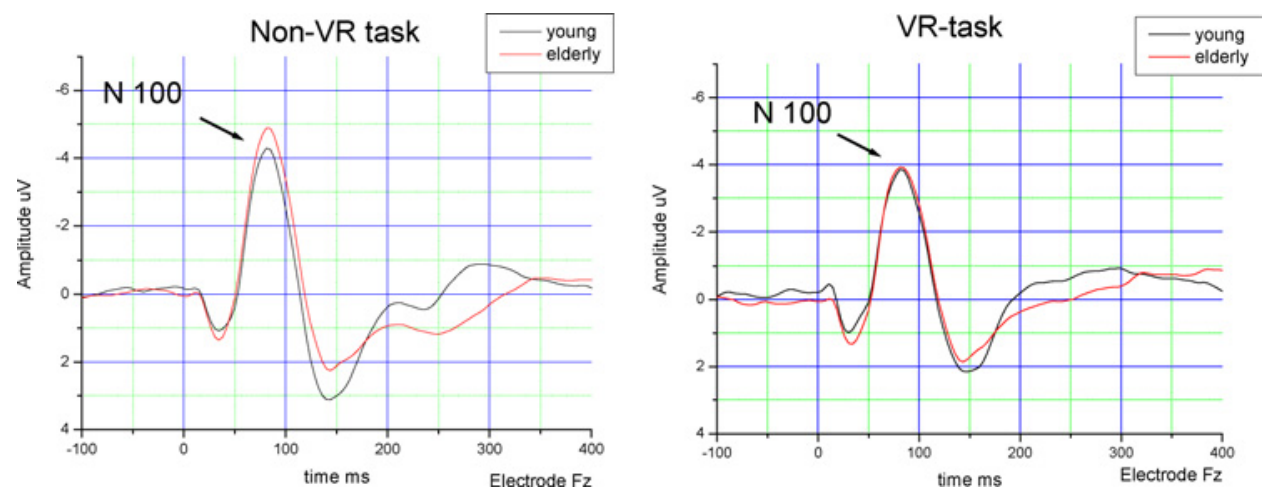

Fig. 3. Grand average of ERPs to frequent tones at Fz. Comparison between age groups and task condition. 

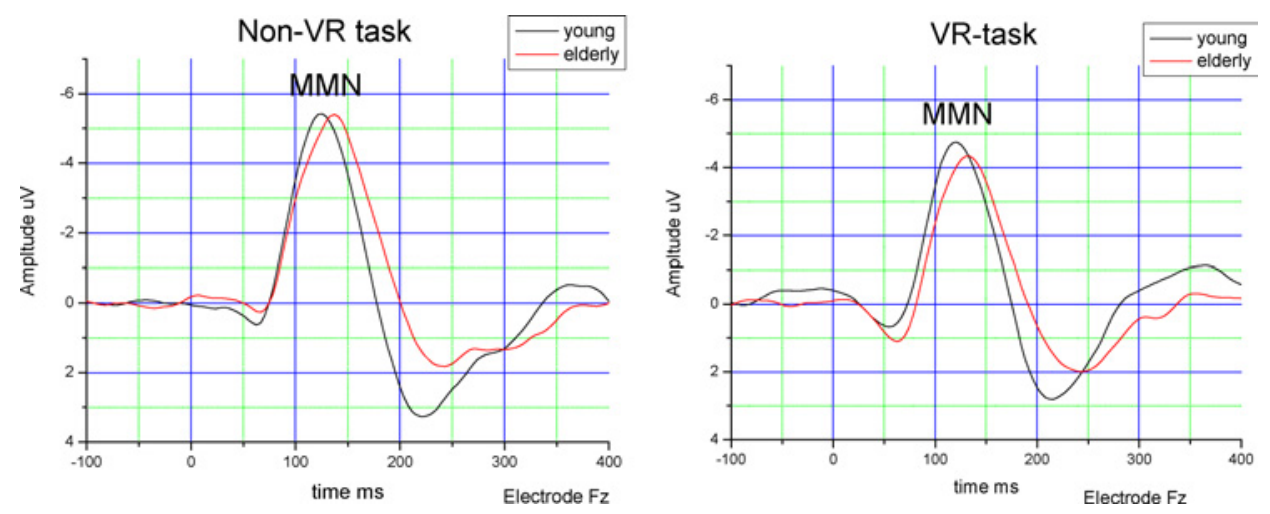

Fig. 4. Grand average of difference curves (responses to rare tones minus responses to frequent tones). Comparison between age groups and task condition.

$p=0.35$ ). The same was true for Condition II (latency Fz: young, mean $=92.3 \pm 10.25 \mathrm{~ms}$; old, mean $=89.40 \pm 7.49 \mathrm{~ms} ; F_{1,44}=1.14, p=0.29$; amplitude Fz: young, mean $=$ $-3.85 \pm 1.60 \mu \mathrm{V}$; old, mean $\left.=-4.17 \pm 1.17 \mu \mathrm{V} ; F_{1,44}=0.56, p=0.46\right)$.

In the next step, the direct comparison between VR and non-VR did not reveal any significant difference of the N100 component in both age groups. The data indicate that the processing of the frequent tone as given by N100 is neither changed by the task condition (non-VR/VR) nor by the factor age (young/elderly).

On the other hand, the pre-attentional processing as given by the MMN differed between age groups. Figure 4 illustrates the difference curves (responses to rare tones minus responses to frequent tones) for the young and the elderly group in both task conditions.

In the non-VR condition (left side), there is a delay of the mismatch negativity (MMN, peak latency; Fz) of about $13 \mathrm{~ms}$ between age groups (young, mean $=128.64 \pm 10.99 \mathrm{~ms}$; old, mean $\left.=141.90 \pm 11.57 \mathrm{~ms} ; F_{1,44}=15.842, p<0,0001\right)$. This delay might be indicative for a beginning process, possibly more pronounced in older groups than the chosen groups. In contrast, the amplitudes do not differ in both age groups for each condition (statistical data not shown). Interestingly, the observed slight delay of the MMN is reproduced during the "VR-condition." During the VR performance the peak amplitude is also delayed ( $p<0.05$ ) in the elderly group (about $10 \mathrm{~ms}$, electrodes F3; C3; P3; T4). The delay corresponds to the delay of the non-VR condition $(\mathrm{Fz}$, young, mean $=124.80 \pm$ $12.16 \mathrm{~ms}$; old, mean $=134.40 \pm 17.07 \mathrm{~ms} ; F_{1,44}=4.832, p=0.033$ ).

Figure 5 uses the same traces as in Fig. 4, but now a direct comparison between both task conditions is possible separately for each age group. The presumably more stressful "VR" condition induced an acceleration of the MMN peak latency that only reached significance in the elderly group. Secondly, the amplitude of the MMN is significantly reduced in the elderly group in the VR condition (Fz, young, mean difference $=0.42 \pm$ $1.86 \mu \mathrm{V}, p=0.29$; old mean difference $=0.957 \pm 1.5 \mu \mathrm{V}, p=0.01$ ). Another interesting finding was the acceleration of the repolarisation after the MMN/P3a complex (note arrows in Fig. 3) which might reflect a faster reorientation after distraction by the rare stimulus. This effect can be observed in both age groups. 

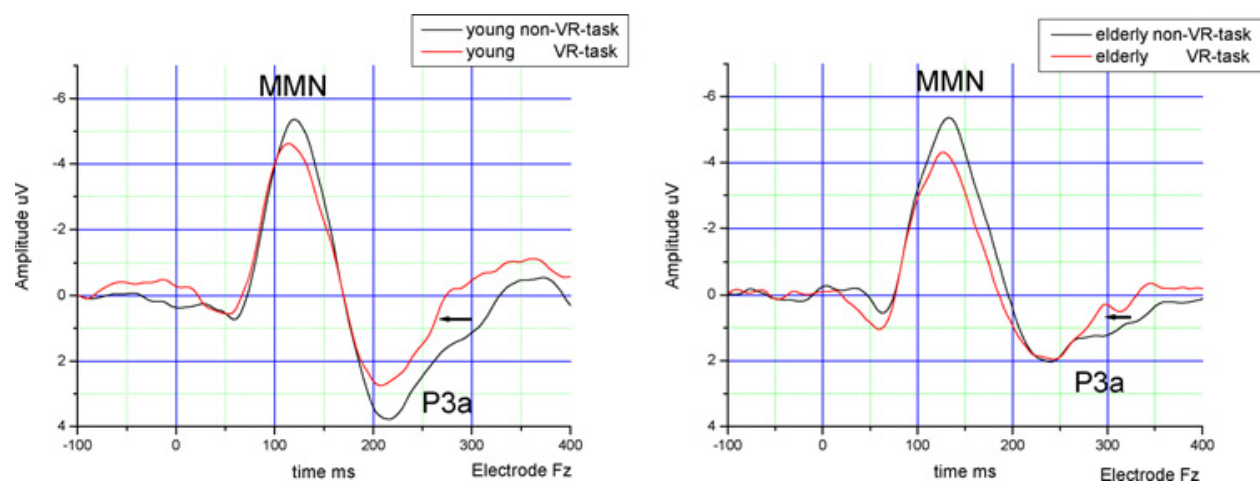

Fig. 5. Grand average of difference curves: comparison between task conditions separately for each age group (left side: younger subjects; right side: elderly subjects). Note the accelerated repolarisation after the P3a in the VR condition.

\section{DISCUSSION}

In the present study, it could be demonstrated that the processing of task-irrelevant rare auditory stimuli is altered in VE compared to a reality condition. This mainly refers to the decreased MMN amplitude and a faster repolarisation after the MMN/P3a complex. This might correspond to a stronger resource allocation in the VR condition filtering out or inhibiting the processing of unexpected auditory events. This is true for both age groups, nevertheless, the effect is more marked in the elderly group. Interestingly, age differences in the non-VR task (delay of MMN) were not strengthened in the VR condition. It is unlikely that the observed effects are specifically correlated to the VR conditions but there is evidence that performing a task in VR leads to a more complex and occlusive condition. Obviously a potent distracting auditory stimulus is sufficient to cause differences in cognitive processing between non-VR and VR conditions whereas a less distracting stimulus (frequent tone) does not reach this threshold.

\section{ACKNOWLEDGMENT}

This research was supported in part by the EU cofunded project RESPECT (Contract number QLRT-2000-00038).

\section{REFERENCES}

Hasher, L., \& Zacks, R. T. (1988). Working memory, comprehension, and aging: A review and a new view. The Psychology of Learning and Motivation, 22, 122-149.

Näätänen, R., \& Picton, T. (1987). The N1 wave of the human electric and magnetic response to sound: A review and an analysis of the component structure. Psychophysiology, 24, 375-425.

Parasuraman, R., \& Beatty, J. (1980). Brain events underlying detection and recognition of weak sensory signal. Science, 210, 80-83.

Powers, R. E. (1994). Neurobiology of aging. In C. E. Coffey, \& J. L. Cummings (Eds.), Textbook of Geriatric Neuropsychiatry (pp. 41-42). Washington, DC: American Psychiatric Press.

Tiitinen, H., May, P., Reinikainen, K., \& Näätänen, R. (1994). Attentive novelty detection in humans is governed by pre-attentive sensory memory. Nature, 372, 90-92. 